\section{Depresión posparto, un problema de salud pública mundial}

\section{Estimado editor:}

La depresión posparto es un trastorno mental afectivo severo, que afecta hasta al $56 \%$ de las mujeres latinas residentes en México y Estados Unidos durante los primeros 4 meses tras el nacimiento de su bebé. Su incidencia mundial es del 15\% (1), y está incluida en el Manual Diagnóstico y Estadístico de los Trastornos Mentales, quinta edición (DSM-5) bajo la denominación de "trastorno depresivo durante el período perinatal" (2). Se asocia a suicidio y filicidio, y el primero es una de las causas más importantes de muerte materna durante el período perinatal (3).

La depresión posparto se relaciona con síntomas psicológicos y alteración de patrones biológicos, entre los que están un estado emocional triste, altos niveles de cansancio, trastornos del sueño y afectación del eje hipotalámico-hipofisiario-adrenal, responsable de la secreción de la hormona cortisol, necesaria para hacer frente a situaciones de estrés. Aunque los síntomas suelen aparecer tras el nacimiento del bebé, las mujeres que posteriormente desarrollan depresión posparto presentan síntomas psicológicos durante el embarazo (somatización, depresión, ansiedad y estrés específico del embarazo) y mayores niveles de cortisol desde el primer trimestre (4). Las altas tasas de depresión posparto entre mujeres latinoamericanas residentes en distintos países, junto con la mayor cantidad de síntomas psicopatológicos que las mujeres inmigrantes experimentan durante el período posparto, hacen necesaria la creación de estrategias para apoyar y facilitar el acceso de este grupo de riesgo a los servicios de salud mental. A este problema se suma que las mujeres buscan menos ayuda psicológica y médica durante el embarazo y el puerperio y por ese motivo tienen menos información sobre las vías de ayuda que otros grupos de mujeres. Además, durante el período perinatal existe un bajo reconocimiento de la efectividad de los servicios de salud mental (1).

Ante esta situación, sería idóneo promover la investigación en torno al embarazo y el puerperio, facilitando que se incluyan mujeres embarazadas en investigación, como se promueve desde el Foro Global de Bioética en Investigación (5). Además, sería recomendable el cribado de la depresión posparto a toda mujer en las primeras horas tras el nacimiento de su bebé mediante la aplicación de escalas psicológicas de depresión posparto, así como el control de los niveles de estrés durante el embarazo mediante instrumentos psicológicos y la evaluación de los niveles de cortisol en el pelo, que pueden predecir el grupo de embarazadas con riesgo de presentar síntomas de depresión posparto (4).

La aplicación de medidas preventivas en torno a la depresión posparto desde la perspectiva de la salud pública mejorará la salud de las mujeres durante el embarazo y el puerperio, y fortalecerán la salud y el desarrollo fetal e infantil $(1,5)$.

Conflictos de interés. Ninguno declarado por los autores.

Declaración. Las opiniones expresadas en este manuscrito son responsabilidad del autor y no reflejan necesariamente los criterios ni la política de la RPSP/PAJPH y/o de la OPS.

\section{Rafael A. Caparros-Gonzalez Borja Romero-Gonzalez \\ María Isabel Peralta-Ramirez}

Centro de Investigación Mente, Cerebro y Comportamiento (CIMCYC), Universidad de Granada, Granada, España

$\bowtie$ Rafael A. Caparros-Gonzalez, rafawolfy@gmail.com

Forma de citar Caparros-Gonzalez R, Romero-Gonzalez B, PeraltaRamirez MI Depresión posparto, un problema de salud pública mundial [carta]. Rev Panam Salud Publica. 2018;42:97 doi: https://doi. org/10.26633/RPSP.2018.97

\section{REFERENCIAS}

1. Barrera AZ, Nichols AD. Depression help-seeking attitudes and behaviors among an Internet based sample of Spanish-speaking perinatal women. Rev Panam Salud Publica. 2015;37(3):148-53.

2. Degner D. Differentiating between "baby blues," severe depression, and psychosis. BMJ. 2017;359:j4692. doi: https://doi.org/10.1136/ bmj.j4692

3. Gressier F, Guillard V, Cazas O, Falissard B, Glangeaud-Freudenthal NM, Sutter-Dallay AL. Risk factors for suicide attempt in pregnancy and the post-partum period in women with serious mental illnesses. J Psychiatr Res. 2017;84:284-91. doi: 10.1016/j.jpsychires.2016.10.009

4. Caparros-Gonzalez RA, Romero-Gonzalez B, Strivens-Vilchez $\mathrm{H}$, Gonzalez-Perez R, Martinez-Augustin O, Peralta-Ramirez MI. Hair cortisol levels, psychological stress and psychopathological symptoms as predictors of postpartum depression. PloS one. 2017;12(8):e0182817.

5. Saenz C, Alger J, Beca JP, Belizán JM, Cafferata ML, Canario Guzmán JA et al. Un llamado ético a la inclusión de mujeres embarazadas en investigación. Rev Panam Salud Publica. 2017;41:e13. 